\title{
Superfluid phases of ultracold Fermi gases on a checkerboard superlattice
}

\author{
M. Iskin \\ Department of Physics, Koç University, Rumelifeneri Yolu, 34450 Sartyer, Istanbul, Turkey \\ (Received 2 May 2013; revised manuscript received 28 August 2013; published 7 November 2013)
}

\begin{abstract}
We analyze the ground-state phase diagram of two-component Fermi gases loaded into a two-dimensional checkerboard superlattice, i.e., a double-well optical lattice, potential within the BCS mean-field theory. We show that, by coupling the two $s$-wave sublattice superfluid order parameters, a checkerboard potential gives rise to an effectively two-band model with three (two intraband and an interband) nonlocal order parameters. We study the evolution of these order parameters as a function of particle filling, interaction strength, and checkerboard potential and find that the system always prefers the 0 -phase solutions, i.e., where the phase difference between sublattice order parameters is 0 , but never the $\pi$-phase one. In addition, we find that the ground state of the system undergoes a superfluid-normal quantum phase transition at half fillings beyond a critical checkerboard potential $C$, the threshold of which is precisely determined by the magnitude of the order parameter at $C=0$, and that the normal state rapidly turns into a checkerboard insulator as $C$ increases.
\end{abstract}

DOI: $10.1103 /$ PhysRevA.88.053606

PACS number(s): 03.75.Ss, 05.30.Fk, 03.75.Hh

\section{INTRODUCTION}

Optical superlattices [1-4] have been of interest to the cold-atom community for a long time now, and they have received more interest in the past few years since they may allow for the study of topological phases of matter in the cold-atom context [5-8]. One way to realize a checkerboard superoptical lattice is to superimpose two independent optical standing waves differing in period by a factor of two, e.g., $\lambda / 2$ and $\lambda$, and tunable intensities and relative phases. In particular, a double-well optical lattice can be produced by arranging the shorter-wavelength $(\lambda / 2)$ lattice potential in such a way to split each potential well of the longer-wavelength $(\lambda)$ lattice into two. In addition, the energy difference between the wells of the resultant double-well potential can also be controlled by tuning the relative phase of the optical potentials [1-4].

In spite of serious challenges in producing fermion superfluids in earlier optical lattice experiments [9-13], there is some recent experimental evidence for superfluid, metallic, and insulating phases [14-16] (see also the recent review [17]). Motivated by these experiments. in this paper, we investigate the ground-state phases of two-component Fermi gases loaded into a two-dimensional checkerboard superlattice. For this purpose, we study a Fermi-Hubbard-type lattice model which includes, in addition to the usual nearest-neighbor hopping and onsite (attractive) interaction, an onsite energy difference between sublattice sites, i.e., a staggered checkerboard potential. We note that the the phase diagram of the Bose-Hubbard versions of such a model have recently been studied for the hard-core [18] and soft-core [19] bosons.

Our main findings, within the single-band tight-binding BCS mean-field theory, are as follows. First, we show that the $s$-wave sublattice order parameters, which are momentum independent in the original Hamiltonian, are coupled by the presence of a checkerboard potential, and this gives rise to a two-band model with three (two intraband and an interband) nonlocal (momentum-dependent higher partial waves) order parameters in the basis where the single-particle Hamiltonian is diagonal. We study the evolution of these order parameters as a function of particle filling, interaction strength, and checkerboard potential, and found that the system always prefers the 0-phase solutions, i.e., where the phase difference between sublattice order parameters is 0 , but never the $\pi$-phase one. In addition, we show that the ground state of the system undergoes a superfluid-normal quantum phase transition at half fillings beyond a critical checkerboard potential $C$, the threshold of which is precisely determined by the magnitude of the order parameter at $C=0$, and that the normal state rapidly turns into a checkerboard insulator as $C$ increases.

The rest of the paper is organized as follows. We introduce the single- and many-body Hamiltonians in Sec. II and derive the quasiparticle-hole excitation spectra of the system as well as the complete set of self-consistency (superfluid order parameters and number) equations. We solve the resultant equations in Sec. III and give a detailed analysis of the obtained results mentioned above. A brief summary of our main findings is given in Sec. IV.

\section{HAMILTONIAN}

It is well established that Hubbard-type discrete lattice models can be used to capture the physics of cold atoms loaded into optical lattice potentials [20-25]. For example, much of the theoretical predictions based on the simplest Bose-Hubbard model [26] have been successfully verified with ultracold Bose gases loaded into optical lattices. The prime examples are the realizations of superfluid and Mott insulator phases as well as the transition between the two [20-25]. Motivated by this success, here we study a Fermi-Hubbardtype lattice model to analyze the physics of ultracold Fermi gases loaded into a checkerboard superlattice potential, as described next.

\section{A. Single-particle problem}

Let us first discuss the single-particle problem on a two-dimensional checkerboard superlattice which consists of two interpenetrating square sublattices as illustrated in Fig. 1. Within the tight-binding approximation, the hopping Hamiltonian for such a lattice can be written as $H_{0}=$ $-\sum_{i \in \alpha, j \in \beta, \sigma} t_{i \alpha, j \beta, \sigma} c_{i \alpha \sigma}^{\dagger} c_{j \beta \sigma}$, where $\{\alpha, \beta\}=(A, B)$ labels the sublattices and $\sigma=(\uparrow, \downarrow)$ labels the two components of the 


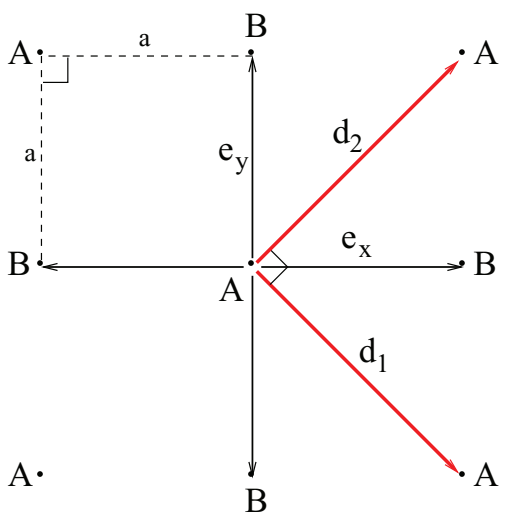

FIG. 1. (Color online) The checkerboard superlattice consisting of interpenetrating $A$ and $B$ square sublattices is sketched. Here, $a$ is the lattice spacing, and $\left(\mathbf{e}_{\mathbf{x}}=a \widehat{\mathbf{x}}, \mathbf{e}_{\mathbf{y}}=a \widehat{\mathbf{y}}\right)$ and $\left(\mathbf{d}_{\mathbf{1}}=\mathbf{e}_{\mathbf{x}}-\mathbf{e}_{\mathbf{y}}\right.$, $\mathbf{d}_{\mathbf{2}}=\mathbf{e}_{\mathbf{x}}+\mathbf{e}_{\mathbf{y}}$ ) are the primitive unit vectors used to construct the single-particle problem.

Fermi gas, $t_{i \alpha, j \beta, \sigma}$ is the tunneling (hopping) amplitude of $\sigma$ fermions between lattice sites $i \alpha$ and $j \beta$, and $c_{i \alpha \sigma}^{\dagger}\left(c_{i \alpha \sigma}\right)$ operator creates (annihilates) a $\sigma$ fermion at lattice site $i \alpha$. In this paper, we limit ourselves to the simplest model where $t_{i \alpha, j \beta, \sigma}=t_{\ell \sigma}$ is nonzero for all nearest-neighbor lattice sites but 0 otherwise. However, we also allow the possibility of alternating hopping elements with a checkerboard pattern where $\ell$ may take two values. In particular, let us assume $t_{1 \sigma}$ $\left(t_{2 \sigma}\right)$ corresponds to the hopping amplitude from the sublattice $A$ to the sublattice $B$ in the positive (negative) $x$ and $y$ directions. We note that the next-nearest-neighbor hoppings are suppressed by an order of magnitude compared to $t_{\ell \sigma}$ in a typical atomic setting [17].

The eigenvalues of such real-space hopping Hamiltonians can be obtained by taking advantage of the discrete translational symmetry and transforming them to the Fourier (momentum) space. Therefore, we assume a single-band description and introduce $c_{i \alpha \sigma}=\left(1 / \sqrt{M_{\alpha}}\right) \sum_{\mathbf{k}_{\alpha} \in 1 \mathrm{BZ}} c_{\mathbf{k}_{\alpha} \alpha \sigma} e^{i \mathbf{k}_{\alpha} \cdot \mathbf{r}_{\mathbf{i}}}$, where $M_{A}=M_{B}=M / 2$ is the number of sublattice sites, $\mathbf{k}_{\alpha}$ is the momentum, $1 \mathrm{BZ}$ is the corresponding first Brillouin zone, and $\mathbf{r}_{\mathbf{i}}$ is the position of the lattice site $i$. Using this transformation, we rewrite the Hamiltonian in momentum space as $H_{0}=\sum_{\mathbf{k} \sigma}\left(\epsilon_{\mathbf{k} \sigma} c_{\mathbf{k} A \sigma}^{\dagger} c_{\mathbf{k} B \sigma}+\right.$ H.c. $)$, where

$$
\begin{aligned}
\epsilon_{\mathbf{k} \sigma}= & -2\left(t_{1 \sigma}+t_{2 \sigma}\right) \cos \left(k_{1} d / 2\right) \cos \left(k_{2} d / 2\right) \\
& -2 i\left(t_{1 \sigma}-t_{2 \sigma}\right) \cos \left(k_{1} d / 2\right) \sin \left(k_{2} d / 2\right)
\end{aligned}
$$

is in general a complex number. Here, $k_{i}=\mathbf{k} \cdot \mathbf{d}_{\mathbf{i}} / d$ corresponds to the projections of the momentum vector along the $\mathbf{d}_{\mathbf{i}}$ directions (see Fig. 1), where $d=\sqrt{2} a, c_{\mathbf{k} \alpha \sigma}^{\dagger}\left(c_{\mathbf{k} \alpha \sigma}\right)$ operator creates (annihilates) a $\sigma$ fermion with momentum $\mathbf{k} \equiv\left(k_{x}, k_{y}\right)$ on $\alpha$ sublattice, and H.c. is the Hermitian conjugate. Thus, the single-particle dispersion relations are $\varepsilon_{\mathbf{k} \sigma r}=r\left|\epsilon_{\mathbf{k} \sigma}\right|$, where $r=(+,-)$ labels the two bands. Note that, since the translational symmetry is doubled in real space, the original $\mathbf{k}$ space is halved but the number of bands is doubled in such a way that this dispersion recovers the usual result, i.e., $\varepsilon_{\mathbf{k} \sigma}=-2 t_{\sigma}\left[\cos \left(k_{x} a\right)+\cos \left(k_{y} a\right)\right]$, in the absence of a sublattice structure when $t_{1 \sigma}=t_{2 \sigma}=t_{\sigma}$.
In addition to the hopping part, we include an onsite checkerboard lattice potential, i.e., an alternating energy offset between sublattices, which is given by $H_{c b}=-C \sum_{i \in \alpha, \sigma} \gamma_{\alpha} c_{i \alpha \sigma}^{\dagger} c_{i \alpha \sigma}$, where $C \geqslant 0$ is its strength and $\gamma_{\alpha}=+1(-1)$ for $\alpha=A(B)$. This term lowers (raises) the onsite energy of the $A(B)$ sublattice sites by $C$. Using the Fourier transformation described above, we rewrite the total Hamiltonian $H_{C}=H_{0}+H_{c b}$ in $\mathbf{k}$ space and diagonalize it, leading to $H_{C}=\sum_{\mathbf{k} \alpha \sigma} \varepsilon_{\mathbf{k} \sigma \alpha} b_{\mathbf{k} \alpha \sigma}^{\dagger} b_{\mathbf{k} \alpha \sigma}$, where $\varepsilon_{\mathbf{k} \sigma \alpha}=\gamma_{\alpha} \sqrt{\left|\epsilon_{\mathbf{k} \sigma}\right|^{2}+C^{2}}$ are the quasiparticle-hole dispersion relations in the presence of a checkerboard potential and $b_{\mathbf{k} \alpha \sigma}^{\dagger}$ $\left(b_{\mathbf{k} \alpha \sigma}\right)$ operator is the new quasiparticle creation (annihilation) operator in the transformed basis. Note that filling the system halfway (a pair of $\uparrow$ and $\downarrow$ particles per two lattice sites) yields a band insulator phase as long as $C \neq 0$, and that $\varepsilon_{\mathbf{k} \sigma \alpha}$ reduces to $\varepsilon_{\mathbf{k} \sigma r}$ when $C=0$ as expected. We note that the $c_{\mathbf{k} \alpha \sigma}$ and $b_{\mathbf{k} \alpha \sigma}$ operators are related via a Bogoliubov transformation $c_{\mathbf{k} A \sigma}=u_{\mathbf{k} \sigma A} b_{\mathbf{k} A \sigma}+u_{\mathbf{k} \sigma B} b_{\mathbf{k} B \sigma}$ and $c_{\mathbf{k} B \sigma}=v_{\mathbf{k} \sigma A} b_{\mathbf{k} A \sigma}+$ $v_{\mathbf{k} \sigma B} b_{\mathbf{k} B \sigma}$, where $u_{\mathbf{k} \sigma \alpha}$ and $v_{\mathbf{k} \sigma \alpha}$ are the components of the eigenvector that corresponds to the eigenvalue $\varepsilon_{\mathbf{k} \sigma \alpha}$. The eigenvectors are orthonormal in such a way that $u_{\mathbf{k} \sigma \alpha} / v_{\mathbf{k} \sigma \alpha}=$ $\epsilon_{\mathbf{k} \sigma} /\left(C+\varepsilon_{\mathbf{k} \sigma \alpha}\right)$. Having discussed the single-particle Hamiltonian, next we move on to the many-particle problem.

\section{B. Many-particle problem}

For the many-particle problem, the effects of local (onsite) and attractive interparticle density-density interactions can be taken into account within the BCS meanfield approximation, which is known to work well for weak interactions at all temperatures and even for moderate interactions at $T=0$ [27-29]. For this purpose, we introduce sublattice-dependent superfluid order parameters $\Delta_{A}$ and $\Delta_{B}$ with $s$-wave symmetry, as defined by $\Delta_{\alpha}=$ $-(2 g / M) \sum_{i \in \alpha}\left\langle c_{i \alpha \downarrow} c_{i \alpha \uparrow}\right\rangle$ in real or $-(2 g / M) \sum_{\mathbf{k}}\left\langle c_{-\mathbf{k} \alpha \downarrow} c_{\mathbf{k} \alpha \uparrow}\right\rangle$ in $\mathbf{k}$ space, where $g \geqslant 0$ is the strength of the interaction and $\langle\cdots\rangle$ is a thermal average. Therefore, the interaction contribution to the $\mathbf{k}$-space Hamiltonian can be written as [30] $H_{m f}=(M / g) \sum_{\alpha}\left|\Delta_{\alpha}\right|^{2} / 2+\sum_{\mathbf{k} \alpha}\left(\Delta_{\alpha}^{*} c_{-\mathbf{k} \alpha \downarrow} c_{\mathbf{k} \alpha \uparrow}+\right.$ H.c. $)$. We also introduce a spin-dependent chemical potential $\mu_{\sigma}$ term to the Hamiltonian, i.e., $H_{\mu}=-\sum_{i \in \alpha, \sigma} \mu_{\sigma} c_{i \alpha \sigma}^{\dagger} c_{i \alpha \sigma}$ in real or $-\sum_{\mathbf{k} \alpha \sigma} \mu_{\sigma} c_{\mathbf{k} \alpha \sigma}^{\dagger} c_{\mathbf{k} \alpha \sigma}$ in $\mathbf{k}$ space, which allows us to fix the number of $\sigma$ fermions independently of each other.

Thus, the total many-body mean-field Hamiltonian $H=H_{0}+H_{c b}+H_{m f}+H_{\mu}$ for the checkerboard superlattice can be compactly written in $\mathbf{k}$ space as $H=$ $(M / g) \sum_{\alpha}\left|\Delta_{\alpha}\right|^{2} / 2-2 \sum_{\mathbf{k}} \mu_{\downarrow}+\sum_{\mathbf{k}} \psi_{\mathbf{k}}^{\dagger} \mathbf{D}_{\mathbf{k}} \psi_{\mathbf{k}}$, where $\psi_{\mathbf{k}}^{\dagger}=$ $\left(c_{\mathbf{k} A \uparrow}^{\dagger}, c_{\mathbf{k} B \uparrow}^{\dagger}, c_{-\mathbf{k} B \downarrow}, c_{-\mathbf{k} A \downarrow}\right)$ denotes the fermionic operators collectively, and the Hamiltonian matrix $\mathbf{D}_{\mathbf{k}}$ is

$\mathbf{D}_{\mathbf{k}}=\left(\begin{array}{cccc}-\mu_{\uparrow}-C & \epsilon_{\mathbf{k} \uparrow} & 0 & \Delta_{A} \\ \epsilon_{\mathbf{k} \uparrow}^{*} & -\mu_{\uparrow}+C & \Delta_{B} & 0 \\ 0 & \Delta_{B}^{*} & \mu_{\downarrow}-C & -\epsilon_{-\mathbf{k} \downarrow} \\ \Delta_{A}^{*} & 0 & -\epsilon_{-\mathbf{k} \downarrow}^{*} & \mu_{\downarrow}+C\end{array}\right)$.

In this paper, we consider equal hoppings for $\uparrow$ and $\downarrow$ fermions, i.e., $t_{\ell \uparrow}=t_{\ell \downarrow}=t_{\ell}$, leading to $\epsilon_{\mathbf{k} \uparrow}=\epsilon_{-\mathbf{k} \downarrow}^{*}=\epsilon_{\mathbf{k}}$. The quasiparticle-hole excitation spectra $E_{\mathbf{k} s}$ of the interacting system are given by the eigenvalues of this Hamiltonian matrix, 
and they can be compactly expressed as

$$
\begin{aligned}
E_{\mathbf{k} s} & =-h+\beta_{s} \sqrt{\varepsilon_{\mathbf{k}}^{2}+\mu^{2}+\sum_{\alpha} \frac{\left|\Delta_{\alpha}\right|^{2}}{2}-\frac{(-1)^{s}}{2} \sqrt{X}} \\
X= & 4\left|\epsilon_{\mathbf{k}}\right|^{2}\left(\left|\Delta_{A}\right|^{2}+\left|\Delta_{B}\right|^{2}-2\left|\Delta_{A} \Delta_{B}\right| \cos \Phi\right)+16 \varepsilon_{\mathbf{k}}^{2} \mu^{2} \\
& +\left(\left|\Delta_{A}\right|^{2}-\left|\Delta_{B}\right|^{2}\right)\left(\left|\Delta_{A}\right|^{2}-\left|\Delta_{B}\right|^{2}+8 \mu C\right),
\end{aligned}
$$

where $s=(1,2,3,4)$ with $\beta_{1}=\beta_{2}=-\beta_{3}=-\beta_{4}=1$ labels the eigenvalues, $h=\left(\mu_{\uparrow}-\mu_{\downarrow}\right) / 2$ is the difference, $\mu=$ $\left(\mu_{\uparrow}+\mu_{\downarrow}\right) / 2$ is the average chemical potential, $\varepsilon_{\mathbf{k}}=$ $\sqrt{\left|\epsilon_{\mathbf{k}}\right|^{2}+C^{2}}$, and $\Phi=\phi_{A}-\phi_{B}$ is the phase difference. Here, we assume $\Delta_{\alpha}=\left|\Delta_{\alpha}\right| e^{i \phi_{\alpha}}$. Note that the particle-hole symmetry of the Hamiltonian implies simultaneous transformation of $A \rightarrow B$ and $\mu \rightarrow-\mu$. Note also that, after setting $\Delta_{A}=$ $\Delta_{B}=\Delta_{0}$ in the $C=0$ limit, this expression recovers the usual result $E_{\mathbf{k} s}=-h+\beta_{s} \sqrt{\left(\left|\epsilon_{\mathbf{k}}\right|-\mu\right)^{2}+\left|\Delta_{0}\right|^{2}}$, which is doubly degenerate since the original $\mathbf{k}$ space is halved.

Using the quasiparticle-hole excitation spectra, we obtain the corresponding mean-field thermodynamic potential $\Omega$ for the total Hamiltonian $H$ as

$$
\begin{aligned}
\Omega= & \frac{M}{2 g} \sum_{\alpha}\left|\Delta_{\alpha}\right|^{2}+T \sum_{\mathbf{k} s} \ln \left[\frac{1+\tanh \left(\frac{\beta_{s} E_{\mathbf{k} s}}{2 T}\right)}{2}\right] \\
& -\frac{1}{2} \sum_{\mathbf{k} s} \beta_{s} E_{\mathbf{k} s}-2 \sum_{\mathbf{k}} \mu,
\end{aligned}
$$

where we set the Boltzmann constant $k_{B}$ to unity. Following the usual procedure, we find the lowest-energy state of the system by minimizing $\Omega$ with respect to the amplitudes $\left|\Delta_{\alpha}\right|$ and phase difference $\Phi$, leading to a set of (three) nonlinearly coupled equations. In addition, we may set the total $n=n_{\uparrow}+n_{\downarrow}$ and imbalance $p=n_{\uparrow}-n_{\downarrow}$ number fillings using the thermodynamic identities $n=-(1 / M) \partial \Omega / \partial \mu$ and $p=-(1 / M) \partial \Omega / \partial h$, where $0 \leqslant n_{\sigma} \leqslant 1$. This procedure leads to a set of (five) self-consistency equations that need to be solved simultaneously, and four of those can be explicitly written as

$$
\begin{gathered}
\frac{\left|\Delta_{A}\right|}{g}=\frac{1}{2 M} \sum_{\mathbf{k} s} \frac{\partial E_{\mathbf{k} s}}{\partial\left|\Delta_{A}\right|} \tanh \left(\frac{E_{\mathbf{k} s}}{2 T}\right), \\
\frac{\left|\Delta_{B}\right|}{g}=\frac{1}{2 M} \sum_{\mathbf{k} s} \frac{\partial E_{\mathbf{k} s}}{\partial\left|\Delta_{B}\right|} \tanh \left(\frac{E_{\mathbf{k} s}}{2 T}\right), \\
n=\frac{1}{2 M} \sum_{\mathbf{k} s}\left[1+\frac{\partial E_{\mathbf{k} s}}{\partial \mu} \tanh \left(\frac{E_{\mathbf{k} s}}{2 T}\right)\right], \\
p=\frac{1}{2 M} \sum_{\mathbf{k} s} \frac{\partial E_{\mathbf{k} s}}{\partial h} \tanh \left(\frac{E_{\mathbf{k} s}}{2 T}\right) .
\end{gathered}
$$

Some of these partial derivatives are long and not particularly illuminating, and therefore none of them are shown. Similar to the expressions above, the remaining (fifth) phase-difference equation can be written as $0=\sum_{\mathbf{k} s}\left(\partial E_{\mathbf{k} s} / \partial \Phi\right) \tanh \left[E_{\mathbf{k} s} /(2 T)\right]$, and since $\partial X / \partial \Phi=$ $8\left|\epsilon_{\mathbf{k}}\right|^{2}\left|\Delta_{A} \Delta_{B}\right| \sin \Phi$, we immediately conclude that $\Phi$ is either 0 or $\pi$. However, our numerical results suggest that the $\pi$-phase solution is never realized for the particular model Hamiltonian that we consider in this paper. Note that these k-space summations over the $1 \mathrm{BZ}$ can be converted into the $\mathbf{k}$-space integrations via $\sum_{\mathbf{k}} f\left(k_{1}, k_{2}\right) \equiv$ $\left[M /\left(8 \pi^{2}\right)\right] \int_{-\pi}^{\pi} \int_{-\pi}^{\pi} f(x / d, y / d) d x d y$.

Before attacking this problem via numerical means, we would like to gain some physical insight. For this purpose, we rewrite the mean-field Hamiltonian $H_{m f}$ in the basis of $b_{\mathbf{k} A \sigma}$ and $b_{\mathbf{k} B \sigma}$ operators, i.e., the one where the single-particle checkerboard Hamiltonian is diagonal. Up to a constant term, the resultant four terms can be compactly written as $\sum_{\mathbf{k} \alpha \beta}\left(F_{\mathbf{k} \alpha \beta} b_{-\mathbf{k} \alpha \downarrow} b_{\mathbf{k} \beta \uparrow}+\right.$ H.c. $)$, where the coefficients are given by $F_{\mathbf{k} \alpha \beta}=\Delta_{A}^{*} u_{-\mathbf{k} \downarrow \alpha} u_{\mathbf{k} \uparrow \beta}+\Delta_{B}^{*} v_{-\mathbf{k} \downarrow \alpha} v_{\mathbf{k} \uparrow \beta}$. It is easy to show that the intraband coefficients $F_{\mathbf{k} \alpha \neq \beta}$ vanish when $C \rightarrow 0$ as one may expect. This immediately reveals that $H_{m f}$ has the form of a two-band model Hamiltonian as long as $C \neq 0$. Note that the coefficients $F_{\mathbf{k} A A}, F_{\mathbf{k} B B}$, and $F_{\mathbf{k} A B}$ correspond, respectively, to the k-dependent $A$ intraband, $B$ intraband, and $A B$ interband superfluid order parameters, and all of them are spin singlet with even parity, i.e., $F_{\mathbf{k} \alpha \beta}=F_{-\mathbf{k} \alpha \beta}$, as one may expect. Therefore, the starting $s$-wave sublattice order parameters $\Delta_{A}$ and $\Delta_{B}$, which are $\mathbf{k}$ independent in the original Hamiltonian, are coupled by $C \neq 0$, and this gives rise to three nonlocal (k-dependent higher partial waves) order parameters in the transformed basis. Having derived the self-consistency equations, next we are ready to present main findings of this work mentioned above in the introduction.

\section{NUMERICAL RESULTS}

In this section, we present our numerical results that are obtained by solving Eqs. (6)-(9) for a self-consistent set of $\left|\Delta_{A}\right|,\left|\Delta_{B}\right|$ and $\mu$ as a function of given $n, g$, and $C$ values. Here, we consider only the ground states of populationbalanced Fermi gases and set $T=0$ and $h=0$ [31]. Motivated by the success of earlier theoretical works on the BCS-BEC crossover problem [27-29], we emphasize that the given set of four mean-field equations (that are suitably generalized here to the checkerboard superlattice model) is expected to describe the qualitative physics well for weak interactions at all $T$, and even for moderate and strong interactions at $T=0$, as long as the single-band tight-binding approximation remains valid [33]. In order to isolate the effects of a nonzero $C$ from that of checkerboard hopping, let us first analyze the uniform hopping $t_{1}=t_{2}=t$ case.

\section{A. Uniform hopping: $t_{1}=t_{2}=t$}

In Fig. 2, we set $g=5 t$ and show $\left|\Delta_{A}\right|$ and $\left|\Delta_{B}\right|$ as a function of $n$ for a set of $C$ values. When $C=0,\left|\Delta_{A}\right|=\left|\Delta_{B}\right|$ is symmetric around half filling $(n=1$ or $\mu=0)$, which is a consequence of the particle-hole symmetry of the parent Hamiltonian, and its maximum value at half filling is a consequence of the lattice density of states effect. As mentioned in Sec. II, when $C \neq 0$, the particle-hole symmetry implies simultaneous transformation of $A \rightarrow B$ and $\mu \rightarrow-\mu$ (or $n \rightarrow 2-n)$. This is clearly illustrated in all of our numerical results, and therefore, it is sufficient to restrict our discussion only to low (particle) fillings $0 \leqslant n \leqslant 1$. We note that while $C \neq 0$ increases $\left|\Delta_{A}\right|$ for low fillings as a function of $C$, it decreases $\left|\Delta_{B}\right|$. This is because since $C>0$ lowers (raises) the onsite energy of the $A(B)$ sublattice, the local chemical potential of the $A(B)$ sublattice is also lowered (raised) by $C$. 
(a)

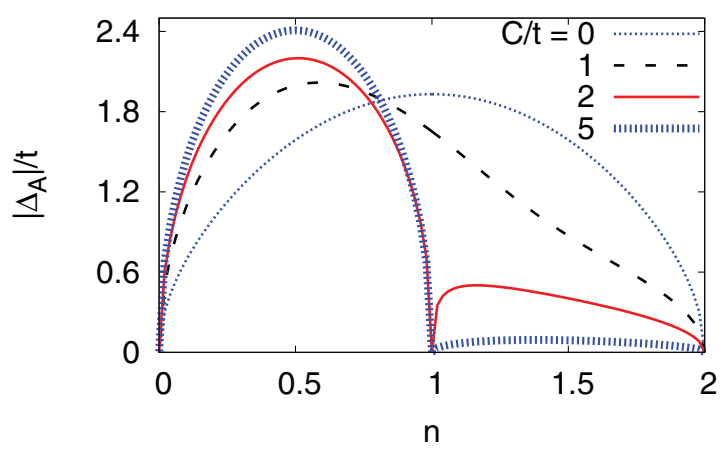

(b)

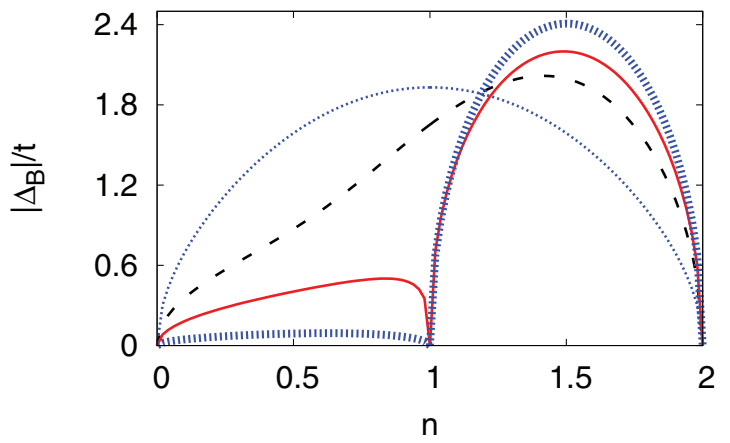

FIG. 2. (Color online) The superfluid order parameters $\left|\Delta_{A}\right|$ in panels (a) and $\left|\Delta_{B}\right|$ in (b) (both in units of hopping $t$ ) are shown as a function of total number filling $n$ for a set of checkerboard potentials $C$ when the interaction strength is $g=5 t$. The particle-hole symmetry implies simultaneous transformation of $A \rightarrow B$ and $\mu \rightarrow-\mu$ (or $n \rightarrow 2-n)$.

Therefore, the $A(B)$ sublattice is effectively becoming more strongly (weakly) interacting as a function of $C$. This effect is clearly seen in Fig. 3(a), where we plot $\left|\Delta_{A}\right|$ and $\left|\Delta_{B}\right|$ as a function of $C$ for a set of $n$ values. In the $C \gg t$ limit, we expect $\left|\Delta_{A}\right|=g \sqrt{n(1-n)}$ and $\left|\Delta_{B}\right|=0$ for $0 \leqslant n \leqslant 1$, which is in perfect agreement with our numerical results.

In addition to these findings, we find that at precisely the half filling the system undergoes a superfluid-normal quantum phase transition beyond a critical $C$, as illustrated in Fig. 3(a). Note that the normal phase at half filling corresponds to a band insulator as discussed in Sec. II A. To gain intuitive understanding of this transition, we analyze the quasiparticle-hole excitation spectra of the interacting system at $n=1$ and therefore set $\mu=0$ and $\left|\Delta_{A}\right|=\left|\Delta_{B}\right|=|\Delta|$ in Eqs. (3) and (4). This gives $X=0$ and a doubly degenerate $E_{\mathbf{k} s}=\beta_{s} \sqrt{\left|\varepsilon_{\mathbf{k}}\right|^{2}+|\Delta|^{2}}$, whose form is the same as the $C=0$ spectra if we identify $\left|\Delta_{C}\right|^{2}=|\Delta|^{2}+C^{2}$. Here, we recall that the quasiparticle-hole dispersion relation of the noninteracting $(g=0)$ system is simply given by $\varepsilon_{\mathbf{k} \alpha}=\gamma_{\alpha} \sqrt{\left|\epsilon_{\mathbf{k}}\right|^{2}+C^{2}}$ (see Sec. II A). Therefore, these results suggest that the system does not favor superfluidity and the normal state with $|\Delta|=0$ becomes the ground state when $C \geqslant\left|\Delta_{0}\right|$, where $\left|\Delta_{0}\right|$ is the $C=0$ value. Our numerical results shown in Fig. 3(b) are consistent with this analysis, where the the critical $C$ values exactly coincide with $\left|\Delta_{0}\right|$ along the phase transition boundary. Given that $\left|\Delta_{0}\right|=\left(g / 2-4 t^{2} / g\right) \sqrt{n(2-n)}$ in the (a)

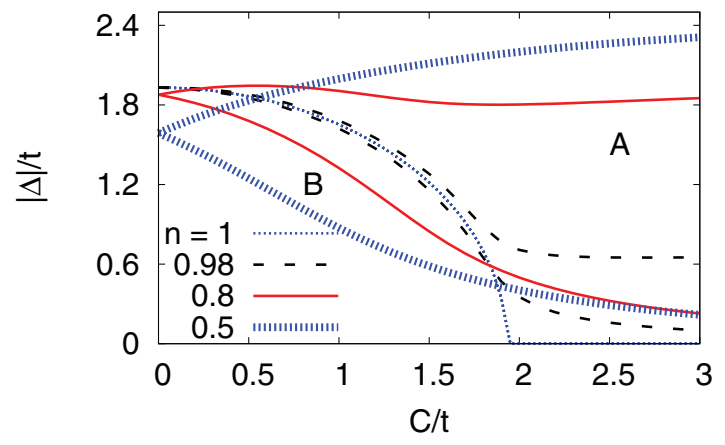

(b)

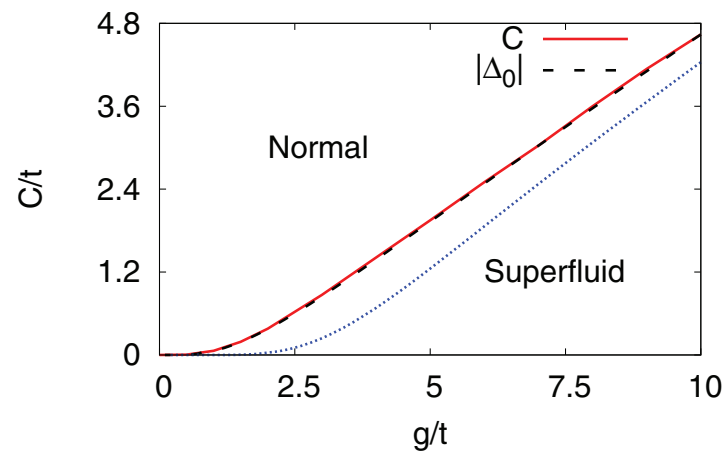

FIG. 3. (Color online) (a) The order parameters $\left|\Delta_{A}\right|$ and $\left|\Delta_{B}\right|$ (in units of hopping $t$ ) are shown as a function of the checkerboard potential $C$ (in units of $t$ ) for a set of total number fillings $n$ when $g=5 t$. (b) The superfluid-normal phase transition boundary is shown as a function of the interaction strength $g$ and $C$ at half filling $(n=1)$. Note that $C=\left|\Delta_{0}\right|$ coincides with the transition boundary, where $\left|\Delta_{0}\right|$ is the magnitude of the order parameter at $C=0$. The normal state rapidly turns into a checkerboard insulator as $C / t \rightarrow \infty$, which is not shown in the figure but discussed in Sec. III A. See Sec. III B for the blue (gray) dotted line.

strong-coupling $(g \gg t)$ limit, by setting $n=1$, we obtain $C=g / 2-4 t^{2} / g$ as the asymptotic limit of the boundary, and this is in perfect agreement with our numerical results. When the critical $C \rightarrow g / 2 \gg t$, we find that the $A(B)$ sublattice has $n_{A} \rightarrow 2\left(n_{B} \rightarrow 0\right)$ so that it corresponds to a sublattice band insulator (fully empty sublattice band), forming a checkerboard insulator. This intuitive result can also be obtained by noting that $n_{A}-n_{B}=-(2 / M) \partial \Omega / \partial C=$ $\sum_{\mathbf{k} s}\left(\partial E_{\mathbf{k} s} / \partial C\right) \tanh \left[\left(E_{\mathbf{k} s} /(2 T)\right]\right.$, which reduces to $n_{A}-$ $n_{B}=(4 / M) \sum_{\mathbf{k}} C / \sqrt{C^{2}+|\Delta|^{2}+\left|\epsilon_{\mathbf{k}}\right|^{2}}$ at half-filling at zero temperature. Therefore, the ground state of the half-filled system first changes from a superfluid to normal when $C=$ $\left|\Delta_{0}\right|$, at which point $|\Delta|$ vanishes, and then the normal state rapidly turns into a checkerboard insulator as $C / t \rightarrow \infty$. For instance, $n_{A}-n_{B}$ becomes $1.9,1.95,1.99$, and 1.999 when $C / t$ is approximately set to $4.9,7.4,17$, and 55 , respectively, in the normal state. Since these numbers are independent of $g / t$, the phase transition is almost (up to $1 \%$ deviation) directly from the superfluid to a checkerboard insulator when $g / t \gtrsim 34$.

We note in passing that while having a nonzero $C$ leads to a staggered pattern not only in the sublattice order parameters, i.e., $\left|\Delta_{A}\right| \neq\left|\Delta_{B}\right|$, but also in the sublattice number fillings, i.e., $n_{A} \neq n_{B}$, we avoid calling the ground states of the system 
a supersolid (when $\left|\Delta_{A}\right| \neq\left|\Delta_{B}\right|$ and $n_{A} \neq n_{B}$ ) or a chargedensity-wave insulator (when $\left|\Delta_{A}\right|=\left|\Delta_{B}\right|=0$ and $n_{A} \neq n_{B}$ is an integer number). This is because since $C \neq 0$ breaks the translational invariance of the lattice, directly causing such an alternating order parameter and filling patterns, we believe it is important to distinguish our superfluid and checkerboard insulator phases from the true supersolid and charge-densitywave insulator ones, for both of which the translational invariance is broken spontaneously due, for instance, to the presence of nearest-neighbor interactions.

\section{B. Checkerboard hopping: $t_{1} \neq t_{2}$}

Before we present our concluding remarks, here we discuss the possibility of having alternating hopping amplitudes. Equation (1) indicates that the effects of small deviations from the uniform hopping, i.e., when $\left|t_{1}-t_{2}\right| \ll t_{1}+t_{2}$, can be taken into account (to a very good approximation) via changing the normalization factor $t$ that is used in the previous section to $\left(t_{1}+t_{2}\right) / 2$. However, in the asymptotic $t_{2} \rightarrow 0$ limit, Eq. (1) gives $\left|\epsilon_{\mathbf{k}}\right|=2 t_{1}\left|\cos \left(k_{1} d / 2\right)\right|$, and one may expect major quantitative differences. For instance, the superfluid-normal phase transition boundary $2 C / t_{1}$ of a half-filled system is shown in Fig. 3(b) as a function of $2 g / t_{1}$ (dotted line), and it is clearly shown that the phase boundary deviates substantially from that of the uniform hopping case. We again note that the normal state rapidly turns into a checkerboard insulator as $C / t_{1} \rightarrow \infty$, for which $n_{A}-n_{B}$ becomes $1.9,1.95,1.99$, and 1.999 when $C / t_{1}$ is approximately set to $4.2,6.1,14$, and 45 , respectively, in the normal state. Having discussed the numerical results, next we briefly summarize the main findings of this paper.

\section{CONCLUSIONS}

To summarize, here we studied the ground-state phases of Fermi gases loaded into a two-dimensional checkerboard superlattice potential, i.e., a double-well optical lattice, consisting of two interpenetrating square sublattices $A$ and $B$. We described this system with a Fermi-Hubbard-type lattice model which includes, in addition to the usual nearest-neighbor hopping $t$ and onsite (attractive) density-density interaction $g$, a sublattice-dependent local (onsite) energy $C$. Within the single-band tight-binding BCS mean-field theory, we reached the following conclusions for such a Hamiltonian. First, we showed that the $s$-wave sublattice order parameters $\Delta_{A}=\left|\Delta_{A}\right| e^{i \phi_{A}}$ and $\Delta_{B}=\left|\Delta_{B}\right| e^{i \phi_{B}}$, which are $\mathbf{k}$ independent in the original Hamiltonian, are coupled by the presence of a checkerboard potential $C \neq 0$, and this gives rise to a two-band model with three (two intraband and an interband) nonlocal (k-dependent higher partial waves) order parameters in the basis where the single-particle Hamiltonian is diagonal. We studied the evolution of these order parameters as a function of particle filling, interaction strength, and checkerboard potential, and found that the system always prefers the 0 -phase $\left(\phi_{A}=\phi_{B}\right)$ solutions but never the $\pi$-phase $\left(\phi_{A}=\phi_{B}+\pi\right)$ one. In addition, we found at precisely half fillings that the ground state of the system undergoes a superfluid-normal quantum phase transition beyond a critical $C$, the threshold of which is precisely determined by the magnitude of the order parameter at $C=0$, and that the normal state rapidly turns into a checkerboard insulator as $C$ increases. One may extend this work in many ways, and motivated by the ongoing experiments [34-38], we are especially interested in studying the effects of artificial gauge fields on the ground-state phases of the system, e.g., the Zeeman field [31] and the so-called optical flux lattices.

\section{ACKNOWLEDGMENTS}

This work is supported by the Marie Curie IRG Grant No. FP7-PEOPLE-IRG-2010-268239, TÜBITAK Career Grant No. 3501-110T839, and TÜBA-GEBIP.
[1] J. Sebby-Strabley, M. Anderlini, P. S. Jessen, and J. V. Porto, Phys. Rev. A 73, 033605 (2006).

[2] P. J. Lee, M. Anderlini, B. L. Brown, J. Sebby-Strabley, W. D. Phillips, and J. V. Porto, Phys. Rev. Lett. 99, 020402 (2007).

[3] S. Fölling, S. Trotzky, P. Cheinet, M. Feld, R. Saers, A. Widera, T. Müller, and I. Bloch, Nature (London) 448, 1029 (2007).

[4] M. Aidelsburger, M. Atala, S. Nascimbéne, S. Trotzky, Y.-A. Chen, and I. Bloch, Phys. Rev. Lett. 107, 255301 (2011).

[5] G. Möller and N. R. Cooper, Phys. Rev. A 82, 063625 (2010).

[6] F. Gerbier and J. Dalibard, New J. Phys. 12, 033007 (2010).

[7] L.-K. Lim, A. Hemmerich, and C. M. Smith, Phys. Rev. A 81, 023404 (2010).

[8] L.-K. Lim, A. Lazarides, A. Hemmerich, and C. Morais Smith, Phys. Rev. A 82, 013616 (2010).

[9] G. Modugno, M. Modugno, F. Riboli, G. Roati, and M. Inguscio, Phys. Rev. Lett. 89, 190404 (2002).

[10] G. Modugno, F. Ferlaino, R. Heidemann, G. Roati, and M. Inguscio, Phys. Rev. A 68, 011601(R) (2003).
[11] M. Köhl, H. Moritz, T. Stöferle, K. Günter, and T. Esslinger, Phys. Rev. Lett. 94, 080403 (2005).

[12] T. Stöferle, H. Moritz, K. Günter, M. Köhl, and T. Esslinger, Phys. Rev. Lett. 96, 030401 (2006).

[13] S. Ospelkaus, C. Ospelkaus, L. Humbert, K. Sengstock, and K. Bongs, Phys. Rev. Lett. 97, 120403 (2006).

[14] J. K. Chin, D. E. Miller, Y. Liu, C. Stan, W. Setiawan, C. Sanner, K. Xu, and W. Ketterle, Nature (London) 443, 961 (2006).

[15] U. Schneider, L. Hackermüller, S. Will, Th. Best, I. Bloch, T. A. Costi, R. W. Helmes, D. Rasch, and A. Rosch, Science 322, 1520 (2008).

[16] R. Jördens, N. Strohmaier, K. Günter, H. Moritz, and Tilman Esslinger, Nature (London) 455, 204 (2008).

[17] T. Esslinger, Ann. Rev. Condens. Matter. Phys. 1, 129 (2010)

[18] I. Hen and M. Rigol, Phys. Rev. B 80, 134508 (2009); I. Hen, M. Iskin, and M. Rigol, ibid. 81, 064503 (2010).

[19] M. Iskin, Eur. Phys. J. B 85, 76 (2012). 
[20] D. Jaksch, C. Bruder, J. I. Cirac, C. W. Gardiner, and P. Zoller, Phys. Rev. Lett. 81, 3108 (1998).

[21] M. Greiner, O. Mandel, T. Esslinger, T. W. Hänsch, and I. Bloch, Nature (London) 415, 39 (2002).

[22] I. Bloch, J. Dalibard, and W. Zwerger, Rev. Mod. Phys. 80, 885 (2008).

[23] F. Gerbier, S. Trotzky, S. Folling, U. Schnorrberger, J. D. Thompson, A. Widera, I. Bloch, L. Pollet, M. Troyer, B. Capogrosso-Sansone, N. V. Prokof'ev, and B. V. Svistunov, Phys. Rev. Lett. 101, 155303 (2008).

[24] Y.-J. Lin, R. L. Compton, K. Jiménez-García, J. V. Porto, and I. B. Spielman, Nature (London) 462, 628 (2009).

[25] Y.-J. Lin, R. L. Compton, A. R. Perry, W. D. Phillips, J. V. Porto, and I. B. Spielman, Phys. Rev. Lett. 102, 130401 (2009).

[26] M. P. A. Fisher, P. B. Weichman, G. Grinstein, and D. S. Fisher, Phys. Rev. B 40, 546 (1989).

[27] A. J. Leggett, in Modern Trends in the Theory of Condensed Matter (Springer-Verlag, Berlin, 1980), p. 13.

[28] P. Nozieres and S. Schmitt-Rink, J. Low. Temp. Phys. 59, 195 (1985).

[29] M. Inguscio, W. Ketterle, and C. Salomon, Ultra-cold Fermi Gases, Proceedings of the International School of Physics Enrico Fermi, Course CLXIV, Varenna, 2006 (IOS Press, Amsterdam, 2008).

[30] Since the Hartree terms in the particle-hole channel simply shift the chemical potentials with no effect on both the self-consistent solutions for the order parameters and the superfluid-normal phase transition boundary, they are not explicitly introduced here.

[31] For population-imbalanced Fermi gases with $h \neq 0$, in addition to the BCS-type uniform superfluid phases (with zero center-ofmass momentum $\mathbf{Q}=\mathbf{0}$ ) considered here, one may also need to consider the Fulde-Ferrell-Larkin-Ovchinnikov (FFLO)-type nonuniform superfluid phases with finite $\mathbf{Q}$ [32]. However, since the eigenvalues of the Hamiltonian matrix are not algebraic for these states, the self-consistency equations need to be computed fully numerically, including the quasiparticle-hole excitation spectrum and their derivatives. It is expected that the imbalanced case involves transitions from FFLO-type superfluids to normal in addition to the BCS-type superfluid to normal one found here.

[32] The possibility of FFLO-type states in population-imbalanced Fermi gases loaded into optical lattices is addressed in the literature by many groups. See e.g., T. Koponen, J. Kinnunen, J.-P. Martikainen, L. M. Jensen, and P. Törmä, New J. Phys. 8, 179 (2006); Y. L. Loh and N. Trivedi, Phys. Rev. Lett. 104, 165302 (2010).

[33] Applications of single-band tight-binding Hubbard models to describe cold atoms loaded into optical lattice potentials are subject to severe experimental limitations. These models work really well away from a Feshbach resonance when the depth of the optical lattice potential is much deeper than the atom recoil energy, in such a way that all of the energy scales $\max \left\{t_{\sigma}, C, g, T\right\}$ are much smaller than the energy gap between the first and second Bloch bands [17].

[34] Y.-J. Lin, K. Jiménez-García, and I. B. Spielman, Nature (London) 471, 83 (2011).

[35] J. Y. Zhang, S. C. Ji, Z. Chen, L. Zhang, Z. D. Du, B. Yan, G. S. Pan, B. Zhao, Y. J. Deng, H. Zhai, S. Chen, and J. W. Pan, Phys. Rev. Lett. 109, 115301 (2012).

[36] P. Wang, Z.-Q. Yu, Z. Fu, J. Miao, L. Huang, S. Chai, H. Zhai, and J. Zhang, Phys. Rev. Lett. 109, 095301 (2012).

[37] L. W. Cheuk, A. T. Sommer, Z. Hadzibabic, T. Yefsah, W. S. Bakr, and M. W. Zwierlein, Phys. Rev. Lett. 109, 095302 (2012).

[38] P. Hauke, O. Tieleman, A. Celi, C. Ölschläger, J. Simonet, J. Struck, M. Weinberg, P. Windpassinger, K. Sengstock, M. Lewenstein, and A. Eckardt, Phys. Rev. Lett. 109, 145301 (2012). 